\title{
Broad-Spectrum Gene Repression using Scaffold Engineering of Synthetic sRNAs
}

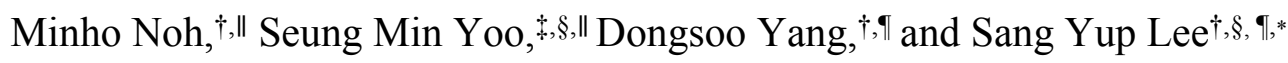

†Department of Chemical and Biomolecular Engineering (BK21 Plus Program), KAIST, 291 Daehak-ro, Yuseong-gu, Daejeon 34141, Republic of Korea

\$School of Integrative Engineering, Chung-Ang University, 84 Heukseok-ro, Dongjak-gu, Seoul 06974, Republic of Korea

§BioProcess Engineering Research Center, KAIST, 291 Daehak-ro, Yuseong-gu, Daejeon 34141, Republic of Korea

『Systems Metabolic Engineering and Systems Healthcare Cross-Generation Collaborative Laboratory, KAIST, 291 Daehak-ro, Yuseong-gu, Daejeon 34141, Republic of Korea

"These authors contributed equally: Minho Noh, Seung Min Yoo.

Corresponding Author

*E-mail: leesy@kaist.ac.kr. Tel: (82) 423503930. 


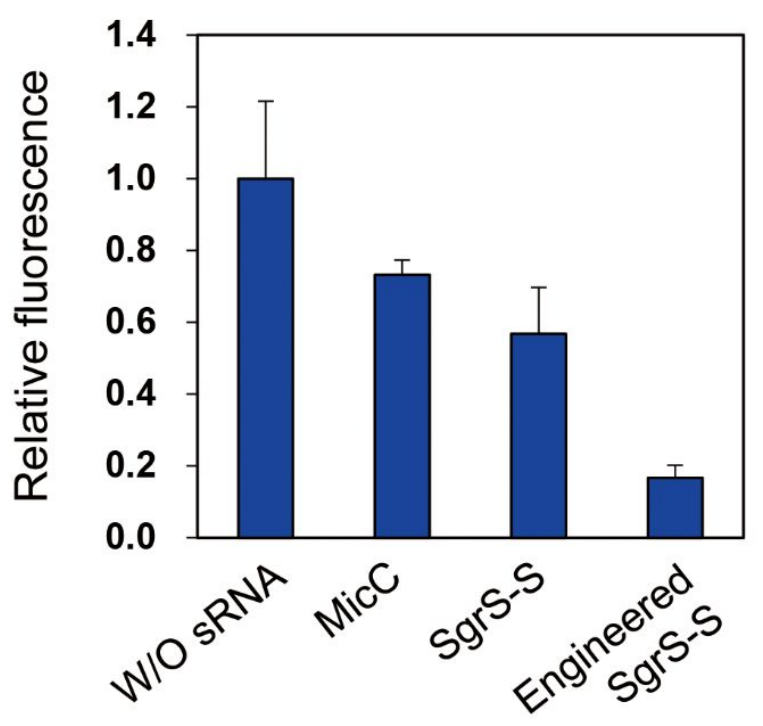

Figure S1. Comparison of anti-DsRed 2 sRNAs derived from various scaffolds. E. coli DH5a cells harboring pACDsRed 2 with anti-DsRed 2 sRNA expressing plasmids (pKK114MDsRed2, pKK114SDsRed2, and pKK114SEDsRed2) were cultivated and the fluorescence intensities were measured. J23114 promoter was used for the expression of sRNAs. The values displayed are average values \pm s.d. $(n=3)$. 


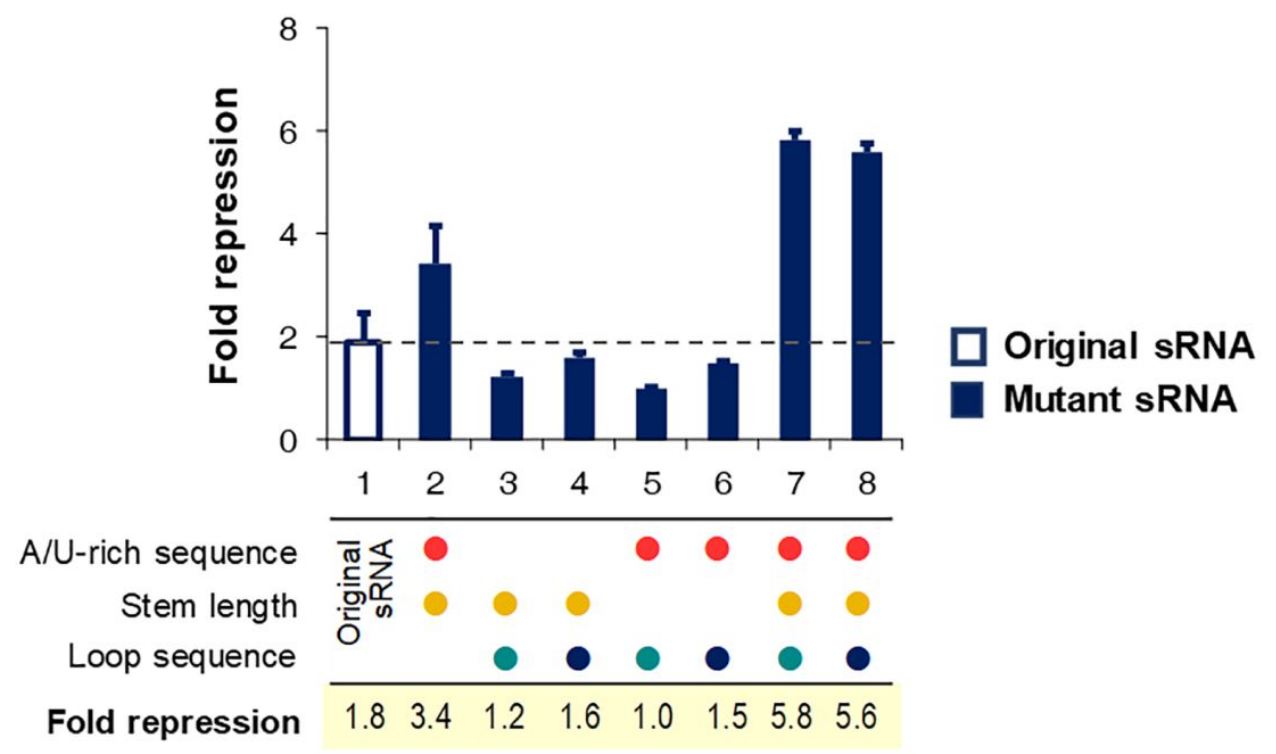

Fold repression

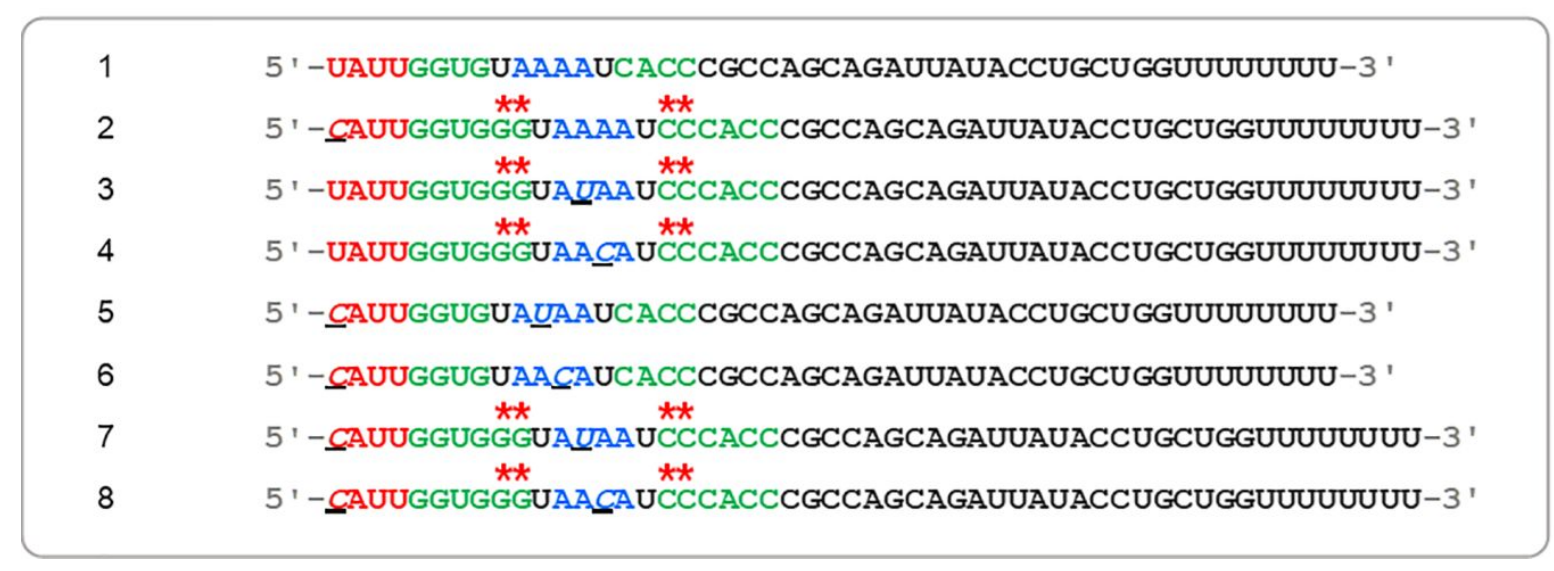

Figure S2. Gene repression activities of sRNAs harboring combinations of the improved modules other than the combinations tested in Figure 2E-F. Nucleotide sequences of the SgrS-S variants are shown in the box below. Texts with asterisks indicate inserted nucleotides. Underlined and italicized texts indicate substituted nucleotides. Data were obtained from three measurements. Fold repression was calculated by dividing the fluorescent intensities of cells without sRNAs by the fluorescent intensities from cells with original or mutant sRNAs (see Figure 1). The values displayed represent average values \pm s.d. $(\mathrm{n}=3)$. 

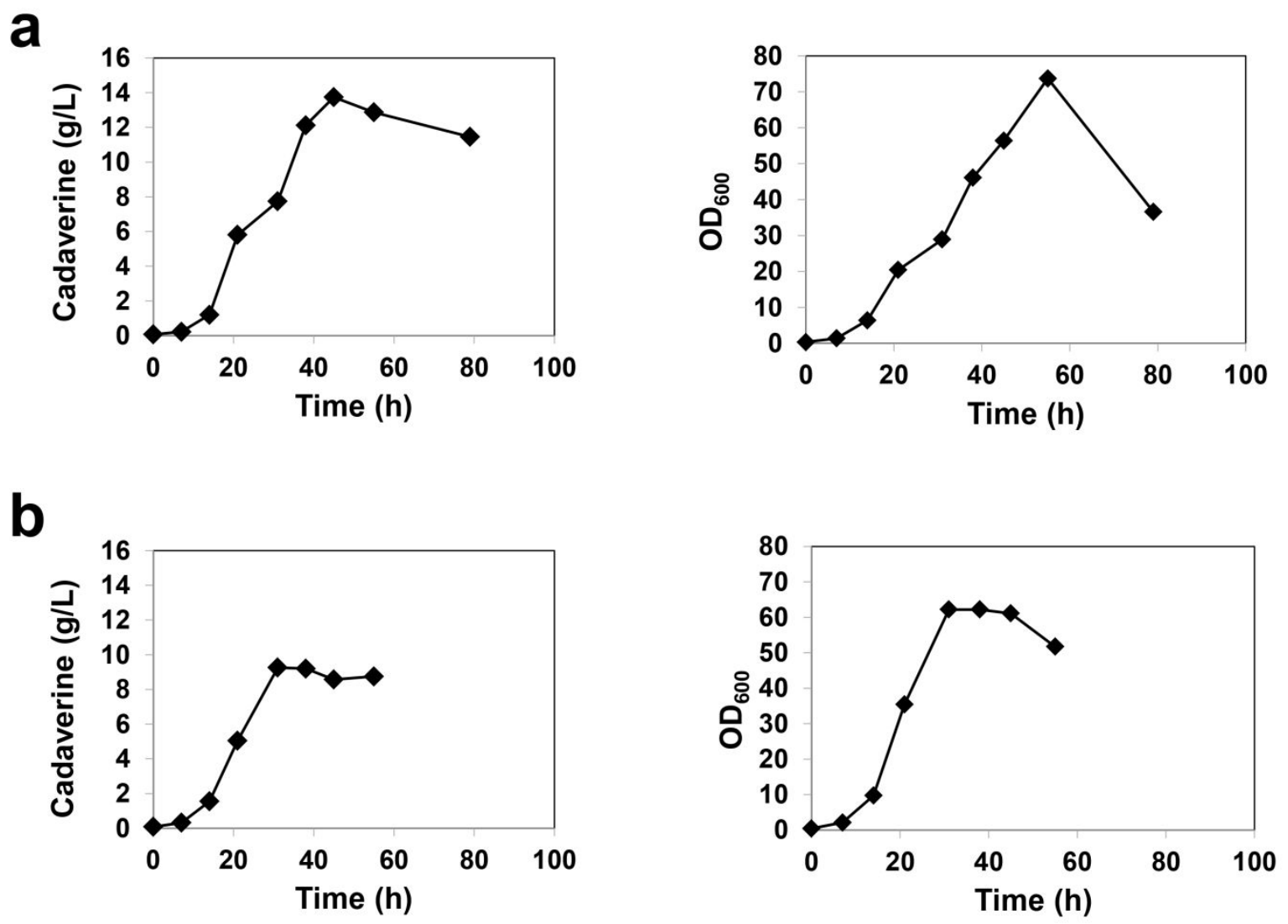

Figure S3. Fed-batch cultures of cadaverine producing strains with expression of improved sRNAs. Each panel indicates the fed-batch cultures result of strain expressing. (A) anti-ser A sRNAs or (B) anti-pgi sRNAs. 